\title{
Existence, positivity and stability for a nonlinear model of cellular proliferation*
}

\author{
Mostafa Adimy $^{\dagger}$ and Fabien Crauste A $^{\ddagger}$ \\ Year 2004 \\ Laboratoire de Mathématiques Appliquées, FRE 2570 \\ Université de Pau et des Pays de l'Adour, \\ Avenue de l'université, 64000 Pau, France
}

\begin{abstract}
In this paper, we investigate a system of two nonlinear partial differential equations, arising from a model of cellular proliferation which describes the production of blood cells in the bone marrow. Due to cellular replication, the two partial differential equations exhibit a retardation of the maturation variable and a temporal delay depending on this maturity. We show that this model has a unique solution which is global under a classical Lipschitz condition. We also obtain the positivity of the solutions and the local and global stability of the trivial equilibrium.
\end{abstract}

Keywords: nonlinear partial differential equation, age-maturity structured model, blood production system, delay depending on the maturity, positivity, local and global stability.

\section{Introduction}

We analyse, in this paper, a mathematical model arising from the blood production system. It is based on a system proposed by Mackey and Rudnicki [19], in 1994, to describe the dynamics of hematopoietic stem cells in the bone marrow. The origin of this system is a model of Burns and Tannock [7] (1970) in which each cell can be either in a proliferating phase or in a resting phase (also called $G_{0}$-phase). The resulting model is a time-age-maturity structured system.

Proliferating cells are in the cell cycle, in that they are committed to divide at the end of the mitosis, the so-called point of cytokinesis. After division, they give birth to two daughter cells which enter immediatly the resting phase. Proliferating cells can also die by apoptosis, a programmed cell death.

The resting phase is a quiescent stage in the cellular development. Cells in this phase can not divide: they mature and, provided they do not die, they enter the proliferating phase and complete the cycle.

The model in [19] has been analysed by Mackey and Rey [17, 18] in 1995, Crabb et al. [8, 9] in 1996, Dyson et al. [10] in 1996 and Adimy and Pujo-Menjouet 3, 4n in 2001 and 2003. In these studies, the authors assumed that all cells divide exactly at the same age.

\footnotetext{
*This paper has been published in Nonlinear Analysis: Real World Applications, 6, 337-366, 2005.

$\dagger$ E-mail: mostafa.adimy@univ-pau.fr

${ }^{\ddagger}$ E-mail: fabien.crauste@univ-pau.fr
} 
However, in the most general situation in a cellular population, it is believed that the time required for a cell to divide is not identical between cells (see Bradford et al. [6]). For example, pluripotent stem cells (which are the less mature cells) divide faster than committed stem cells, which are the more mature stem cells. In 1993, Mackey and Rey [16] considered a model in which the time required for a cell to divide is distributed according to a density, but the authors only made a numerical analysis of their model. Dyson et al. 11, 12, in 2000, considered a time-age-maturity structured equation in which all cells do not divide at the same age. They presented the basic theory of existence, uniqueness and properties of the solution operator. However, in their model, they considered only one phase (the proliferating one), and the intermediary flux between the two phases is not represented. In 2003, Adimy and Crauste [2] considered a model in which the proliferating phase duration is distributed according to a density with compact support. They obtained global stability results for their model.

In this work, we consider the situation when the age at cytokinesis depends on the maturity of the cell at the point of commitment, that means when it enters the proliferating phase. We assume that each cell entering the proliferating phase with a maturity $m$ divides at age $\tau=\tau(m)$, depending on this maturity. This hypothesis can be found, for example, in Mitchison [21] (1971) and John [13] (1981). This yields to the boundary condition (11). To our knowledge, nobody has studied this model, except Adimy and Pujo-Menjouet in [5], where they considered only a linear case.

We obtain a system of first order partial differential equations with a time delay depending on the maturity and a retardation of the maturation variable. We investigate the basic theory of existence, uniqueness, positivity and stability of the solutions of our model.

The paper is organised as follows. In Section 2, we present the time-age-maturity structured model. By using the characteristics method, we reduce this model to a time-maturity structured system, which is formed by two partial differential equations with a time delay depending on the maturity and a nonlocal dependence in the maturity variable. In Section 3, we first give an integrated formulation of our model by using the classical variation of constant formula and then we prove local existence of solutions, by using a fixed-point theorem, and their global continuation. We deduce the global existence. In Section 4 , we obtain the positivity of these solutions by developping a method described by Webb [24. In Section 5 , we concentrate on the stability of the trivial equilibrium of the system and, in the last section, we discuss the model and the asymptotic behaviour.

\section{Biological background and equations of the model}

Each cell is caracterised, in the two phases, by its age and its maturity. The maturity describes the development of the cell. It is the concentration of what composes a cell, such as proteins or other elements one can measure experimentally. The maturity is supposed to be a continuous variable and to range from $m=0$ to $m=1$ in the two phases.

Cells enter the proliferating phase with age $a=0$ and they are committed to undergo cell division a time $\tau$ later, so the age variable ranges from $a=0$ to $a=\tau$ in the proliferating phase. We suppose that proliferating cells can be lost by apoptosis with a rate $\gamma$.

At the cytokinesis age, a cell divides and gives two daughter cells, which enter immediatly the resting phase, with age $a=0$. A cell can stay its entire life in the resting phase, so the age variable ranges from $a=0$ to $a=+\infty$. The resting phase is a quiescent stage in the cellular development. In this phase, cells can either return to the proliferating phase at a rate $\beta$ and complete the cycle or die at a rate $\delta$ before ending the cycle. According to a work of Sachs [22], we suppose that the maturation of a cell and the density of resting cells at a given maturity level determine the capacity of this cell for entering the proliferating phase.

We denote by $p(t, m, a)$ and $n(t, m, a)$ respectively the population densities in the proliferating and the 
resting phases at time $t$, with age $a$ and maturity $m$. The conservation equations are

$$
\begin{gathered}
\frac{\partial p}{\partial t}+\frac{\partial p}{\partial a}+\frac{\partial(V(m) p)}{\partial m}=-\gamma(m) p \\
\frac{\partial n}{\partial t}+\frac{\partial n}{\partial a}+\frac{\partial(V(m) n)}{\partial m}=-(\delta(m)+\beta(m, N(t, m))) n
\end{gathered}
$$

where $V(m)$ is the maturation velocity and $N(t, m)$ is the density of resting cells at time $t$ with a maturity level $m$, defined by

$$
N(t, m)=\int_{0}^{+\infty} n(t, m, a) d a .
$$

We suppose that the function $V$ is continuously differentiable on $[0,1]$, positive on $(0,1]$ and satisfies $V(0)=0$ and

$$
\int_{0}^{m} \frac{d s}{V(s)}=+\infty, \quad \text { for } m \in(0,1]
$$

Since $\int_{m_{1}}^{m_{2}} \frac{d s}{V(s)}$, with $m_{1}<m_{2}$, is the time required for a cell with maturity $m_{1}$ to reach the maturity $m_{2}$, then Condition (3) means that a cell with very small maturity needs a long time to become mature. For example, if

$$
V(m) \underset{m \rightarrow 0}{\sim} \alpha m^{p}, \quad \text { with } \alpha>0 \text { and } p \geq 1,
$$

then Condition (3) is satisfied.

We suppose, throughout this paper, that $\gamma$ and $\delta$ are continuous and non-negative on $[0,1]$. The function $\beta$ is supposed to be positive and continuous.

Equations (1) and (2) are completed by boundary conditions which represent the cellular flux between the two phases. The first condition,

$$
p(t, m, 0)=\int_{0}^{+\infty} \beta(m, N(t, m)) n(t, m, a) d a=\beta(m, N(t, m)) N(t, m),
$$

describes the efflux of cells leaving the resting phase to the proliferating one. Cells entering the proliferating phase with age 0 depend only on the population of the resting phase with a given maturity level.

The second boundary condition determines the transfer of cells from the point of cytokinesis to the resting compartment.

We assume that a cell entering the proliferating phase with a maturity $m \in[0,1]$ divides at age $\tau(m)>0$, and we require that $\tau$ is a continuously differentiable and positive function on $[0,1]$ such that

$$
\tau^{\prime}(m)+\frac{1}{V(m)}>0, \quad \text { for } m \in(0,1]
$$

Since $V(0)=0$, this condition is always satisfied in a neighborhood of the origin. If we suppose that the less mature cells divide faster than more mature cells, that is, if we assume, for example, that $\tau$ is an increasing function, then Condition (5) is also satisfied.

If one consider a cell in the proliferating phase at time $t$, with maturity $m \in(0,1]$, age $a$ and initial maturity (that means at age $a=0) m_{0}$, then, naturally, we have

$$
m_{0} \leq m \quad \text { and } \quad a=\int_{m_{0}}^{m} \frac{d s}{V(s)} \leq \tau\left(m_{0}\right)
$$

If $m$ is the maturity of the cell at the cytokinesis point, then there exists a unique $\Theta(m) \in(0, m)($ the maturity at the point of commitment) such that

$$
\int_{\Theta(m)}^{m} \frac{d s}{V(s)}=\tau(\Theta(m))
$$


because Condition (5) implies that the function

$$
\widetilde{m} \rightarrow \int_{\widetilde{m}}^{m} \frac{d s}{V(s)}-\tau(\widetilde{m})
$$

is continuous and strictly decreasing from $(0, m]$ into $[-\tau(m),+\infty)$. Then, we can define a function $\Theta:(0,1] \rightarrow(0,1]$, where $\Theta(m)$ satisfies $(6)$.

From a biological point of view, $\Theta(m)$ represents the initial maturity of proliferating cells that divide at maturity $m$ (at the point of cytokinesis). Then, from the definition, the age of a cell with maturity $m$ at the point of cytokinesis is $\tau(\Theta(m))$.

Remark that $\Theta$ is continuously differentiable on $(0,1]$ and satisfies

$$
0<\Theta(m)<m, \quad \text { for } m \in(0,1]
$$

This implies, in particular, that

$$
\lim _{m \rightarrow 0} \Theta(m)=0 \quad \text { and } \quad \lim _{m \rightarrow 0} \int_{\Theta(m)}^{m} \frac{d s}{V(s)}=\tau(0)<+\infty .
$$

The property (7) means that cells with null maturity at the point of commitment keep a null maturity in the proliferating phase.

The total number of proliferating cells at time $t$, with maturity $m$, is given by

$$
P(t, m)=\int_{0}^{\tau(\Theta(m))} p(t, m, a) d a .
$$

We consider the characteristic curves $\chi:(-\infty, 0] \times[0,1] \rightarrow[0,1]$, solutions of the ordinary differential equation

$$
\left\{\begin{aligned}
\frac{d \chi}{d s}(s, m) & =V(\chi(s, m)), \quad s \leq 0 \text { and } m \in[0,1], \\
\chi(0, m) & =m .
\end{aligned}\right.
$$

They represent the evolution of cells maturity to reach a maturity $m$ at time 0 from a time $s \leq 0$. They satisfy $\chi(s, 0)=0$ and $\chi(s, m) \in(0,1]$ for $s \leq 0$ and $m \in(0,1]$.

It is not difficult to verify that, if $m \in[0,1]$, then $\Theta(m)$ is the unique solution of the equation

$$
x=\chi(-\tau(x), m) .
$$

At the end of the proliferating phase, a cell with a maturity $m$ divides into two daughter cells with maturity $g(m)$. We assume that $g:[0,1] \rightarrow[0,1]$ is a continuous and strictly increasing function, continuously differentiable on $[0,1)$ and such that $g(m) \leq m$ for $m \in[0,1]$. We also assume, for technical reason and without loss of generality, that

$$
\lim _{m \rightarrow 1} g^{\prime}(m)=+\infty
$$

Then we can set

$$
g^{-1}(m)=1, \quad \text { for } m>g(1)
$$

This means that the function $g^{-1}:[0,1] \rightarrow[0,1]$ is continuously differentiable and satisfies

$$
\left(g^{-1}\right)^{\prime}(m)=0, \quad \text { for } m>g(1) \text {. }
$$

Note that the maturity $m$ of the daughter cells just after division is smaller than $g(1)$. Then, we must have

$$
n(t, m, 0)=0, \quad \text { for } m>g(1) \text {. }
$$


If a daughter cell has a maturity $m$ at birth, then the maturity of its mother at the point of cytokinesis was $g^{-1}(m)$ and, at the point of commitment, it was $\Theta\left(g^{-1}(m)\right)$. We set

$$
\Delta(m)=\Theta\left(g^{-1}(m)\right), \quad \text { for } m \in[0,1] .
$$

From a biological point of view, $\Delta$ gives the link between the maturity of a new born cell and the maturity of its mother at the point of commitment. $\Delta:[0,1] \rightarrow[0,1]$ is continuous, continuously differentiable on $(0,1]$, with $\Delta(0)=0$. Moreover, $\Delta$ is strictly increasing on $(0, g(1))$ with $\Theta(m) \leq \Delta(m)$ and $\Delta(m)=\Theta(1)$ for $m \in[g(1), 1]$.

Then, we can give the second boundary condition,

$$
n(t, m, 0)=2\left(g^{-1}\right)^{\prime}(m) p\left(t, g^{-1}(m), \tau(\Delta(m))\right), \quad \text { for } t \geq 0 \text { and } m \in[0,1] .
$$

One can note that Expression (11) includes also Condition (9).

To complete the description of the model, we specify initial conditions,

$$
p(0, m, a)=\Gamma(m, a), \quad \text { for }(m, a) \in[0,1] \times\left[0, \tau_{\max }\right]
$$

and

$$
n(0, m, a)=\mu(m, a), \quad \text { for }(m, a) \in[0,1] \times[0,+\infty),
$$

where $\tau_{\max }:=\max _{m \in[0,1]} \tau(m)>0$. $\Gamma$ and $\mu$ are assumed to be continuous, and the function

$$
\bar{\mu}: m \mapsto \int_{0}^{+\infty} \mu(m, a) d a
$$

is supposed to be continuous on $[0,1]$.

We put

$$
\xi(t, m):=\exp \left\{-\int_{0}^{t}\left(\gamma(\chi(-s, m))+V^{\prime}(\chi(-s, m))\right) d s\right\},
$$

for $t \geq 0$ and $m \in[0,1]$, and we define the sets

$$
\Omega_{\Delta}:=\{(m, t) \in[0,1] \times[0,+\infty) ; 0 \leq t \leq \tau(\Delta(m))\},
$$

and

$$
\Omega_{\Theta}:=\{(m, t) \in[0,1] \times[0,+\infty) ; 0 \leq t \leq \tau(\Theta(m))\} .
$$

Proposition 2.1. Assume that the initial conditions $\mu$ and $\Gamma$ satisfy, for $m \in[0,1]$,

$$
\Gamma(m, 0)=\beta(m, \bar{\mu}(m)) \bar{\mu}(m) .
$$

Then, the total populations of proliferating and resting cells, $P(t, m)$ and $N(t, m)$, satisfy, for $m \in[0,1]$ and $t \geq 0$,

$$
\begin{aligned}
& \frac{\partial}{\partial t} P(t, m)+\frac{\partial}{\partial m}(V(m) P(t, m))=-\gamma(m) P(t, m)+\beta(m, N(t, m)) N(t, m) \\
& -\left\{\begin{array}{lr}
\pi(m) \xi(t, m) \Gamma(\chi(-t, m), \tau(\Theta(m))-t), & \text { if }(m, t) \in \Omega_{\Theta}, \\
\pi(m) \xi(\tau(\Theta(m)), m) \beta(\Theta(m), N(t-\tau(\Theta(m)), \Theta(m))) \times & \\
N(t-\tau(\Theta(m)), \Theta(m)), & \text { if }(m, t) \notin \Omega_{\Theta},
\end{array}\right.
\end{aligned}
$$




$$
\begin{aligned}
& \frac{\partial}{\partial t} N(t, m)+\frac{\partial}{\partial m}(V(m) N(t, m))=-(\delta(m)+\beta(m, N(t, m))) N(t, m) \\
& + \begin{cases}2\left(g^{-1}\right)^{\prime}(m) \xi\left(t, g^{-1}(m)\right) \Gamma\left(\chi\left(-t, g^{-1}(m)\right), \tau(\Delta(m))-t\right), & \text { if }(m, t) \in \Omega_{\Delta}, \\
\zeta(m) \beta(\Delta(m), N(t-\tau(\Delta(m)), \Delta(m))) N(t-\tau(\Delta(m)), \Delta(m)), & \text { if }(m, t) \notin \Omega_{\Delta},\end{cases}
\end{aligned}
$$

and

$$
\begin{aligned}
P(0, m) & =\bar{\Gamma}(m):=\int_{0}^{\tau(\Theta(m))} \Gamma(m, a) d a \\
N(0, m) & =\bar{\mu}(m),
\end{aligned}
$$

with

and

$$
\pi(m)=\frac{1}{1+V(\Theta(m)) \tau^{\prime}(\Theta(m))}
$$

$$
\zeta(m)=2\left(g^{-1}\right)^{\prime}(m) \xi\left(\tau(\Delta(m)), g^{-1}(m)\right)
$$

Proof. Using (12), (13) and the definitions of $P$ and $N$, we obtain immediatly the equations (18) and (19). System (1)-(2) can be solved by using the method of characteristics. First, we obtain the following representation of solutions of Equation (11),

$$
p(t, m, a)= \begin{cases}\xi(t, m) p(0, \chi(-t, m), a-t), & \text { for } 0 \leq t<a, \\ \xi(a, m) p(t-a, \chi(-a, m), 0), & \text { for } a \leq t .\end{cases}
$$

The initial condition (12) and the boundary condition (4) give

$$
\begin{aligned}
& p(t, m, a)= \\
& \begin{cases}\xi(t, m) \Gamma(\chi(-t, m), a-t), & \text { for } 0 \leq t<a, \\
\xi(a, m) \beta(\chi(-a, m), N(t-a, \chi(-a, m))) N(t-a, \chi(-a, m)), & \text { for } a \leq t .\end{cases}
\end{aligned}
$$

Let $m \in[0,1]$ be given. By integrating Equation (1) with respect to the age, between 0 and $\tau(\Theta(m))$, we obtain

$$
\frac{\partial}{\partial t} P(t, m)+\int_{0}^{\tau(\Theta(m))} \frac{\partial}{\partial m}(V(m) p(t, m, a)) d a=-\gamma(m) P(t, m)+p(t, m, 0)-p(t, m, \tau(\Theta(m))) .
$$

One can note that

$$
\tau^{\prime}(\Theta(m)) \Theta^{\prime}(m) V(m)-1=-\pi(m) .
$$

Since

$$
\frac{\partial}{\partial m}(V(m) P(t, m))=\int_{0}^{\tau(\Theta(m))} \frac{\partial}{\partial m}(V(m) p(t, m, a)) d a+\tau^{\prime}(\Theta(m)) \Theta^{\prime}(m) V(m) p(t, m, \tau(\Theta(m))),
$$

and

$$
\begin{aligned}
& p(t, m, \tau(\Theta(m)))= \\
& \begin{cases}\xi(t, m) \Gamma(\chi(-t, m), \tau(\Theta(m))-t), & \text { if } 0 \leq t<\tau(\Theta(m)) \\
\xi(\tau(\Theta(m)), m) \beta(\chi(-\tau(\Theta(m)), m), N(t-\tau(\Theta(m)), \chi(-\tau(\Theta(m)), m))) \times & \\
N(t-\tau(\Theta(m)), \chi(-\tau(\Theta(m)), m)), & \text { if } \tau(\Theta(m)) \leq t\end{cases}
\end{aligned}
$$


then, using (4) and (8), we obtain Equation (16).

Thanks to (15) and by using the continuity of $\bar{\mu}$, we show that $\lim _{a \rightarrow+\infty} n(t, m, a)=0$.

So, by integrating Equation (2) with respect to the age, between 0 and $+\infty$, it follows that

$$
\frac{\partial}{\partial t} N(t, m)+\frac{\partial}{\partial m}(V(m) N(t, m))=-(\delta(m)+\beta(m, N(t, m))) N(t, m)+n(t, m, 0) .
$$

From the equations $(8)$ and $(10)$, we deduce that

$$
\Delta(m)=\chi\left(-\tau(\Delta(m)), g^{-1}(m)\right) .
$$

Hence, from Equations (11) and (21), we obtain

$$
n(t, m, 0)= \begin{cases}2\left(g^{-1}\right)^{\prime}(m) \xi\left(t, g^{-1}(m)\right) \Gamma\left(\chi\left(-t, g^{-1}(m)\right), \tau(\Delta(m))-t\right), & \text { if }(m, t) \in \Omega_{\Delta}, \\ \zeta(m) \beta(\Delta(m), N(t-\tau(\Delta(m)), \Delta(m))) N(t-\tau(\Delta(m)), \Delta(m)), & \text { if }(m, t) \notin \Omega_{\Delta} .\end{cases}
$$

Equation (17) follows immediatly.

Finally, we can remark that, if $N$ is continuous, Condition (15) implies that the mappings $(m, t) \mapsto$ $F(t, m, N(t-\tau(\Delta(m)), \Delta(m)))$ and $(m, t) \mapsto G(t, m, N(t-\tau(\Theta(m)), \Theta(m)))$, with $F:[0,+\infty) \times[0,1] \times$ $\mathbb{R} \rightarrow \mathbb{R}$ and $G:[0,+\infty) \times[0,1] \times \mathbb{R} \rightarrow \mathbb{R}$ given by

$$
F(t, m, x)= \begin{cases}2\left(g^{-1}\right)^{\prime}(m) \xi\left(t, g^{-1}(m)\right) \Gamma\left(\chi\left(-t, g^{-1}(m)\right), \tau(\Delta(m))-t\right), & \text { if }(m, t) \in \Omega_{\Delta}, \\ \zeta(m) \beta(\Delta(m), x) x, & \text { if }(m, t) \notin \Omega_{\Delta},\end{cases}
$$

and

$$
G(t, m, x)= \begin{cases}\pi(m) \xi(t, m) \Gamma(\chi(-t, m), \tau(\Theta(m))-t), & \text { if }(m, t) \in \Omega_{\Theta}, \\ \pi(m) \xi(\tau(\Theta(m)), m) \beta(\Theta(m), x) x, & \text { if }(m, t) \notin \Omega_{\Theta},\end{cases}
$$

are continuous.

This completes the proof.

One can remark that the solutions of Equations $(17)$ and $(19)$ do not depend on the proliferating population. We extend $N$ by setting

$$
N(t, m)=\bar{\mu}(m), \quad \text { for } t \in\left[-\tau_{\max }, 0\right] \text { and } m \in[0,1]
$$

This extension does not influence our system. However, it will be useful in the following.

\section{Local existence and global continuation}

In this section, we are interested in proving the local existence of an integrated solution of Problem (16)(19). First, we consider an integrated formulation of Problem (16)-(19). We denote by $C[0,1]$ the space of continuous functions on $[0,1]$, endowed with the supremum norm $\|$.$\| , defined by$

$$
\|v\|=\sup _{m \in[0,1]}|v(m)|, \quad \text { for } v \in C[0,1] .
$$

Let us consider the unbounded closed linear operator $A: D(A) \subset C[0,1] \rightarrow C[0,1]$ defined by

$$
D(A)=\left\{u \in C[0,1] ; u \text { differentiable on }(0,1], u^{\prime} \in C(0,1], \lim _{x \rightarrow 0} V(x) u^{\prime}(x)=0\right\}
$$


and

$$
A u(x)= \begin{cases}-\left(\delta(x)+V^{\prime}(x)\right) u(x)-V(x) u^{\prime}(x), & \text { if } x \in(0,1], \\ -\left(\delta(0)+V^{\prime}(0)\right) u(0), & \text { if } x=0 .\end{cases}
$$

Then, we have the following proposition, which characterise the operator $(A, D(A))$.

Proposition 3.1. The operator $A$ is the infinitesimal generator of the strongly continuous semigroup $(T(t))_{t \geq 0}$ defined on $C[0,1]$ by

$$
(T(t) \psi)(x)=K(t, x) \psi(\chi(-t, x)), \quad \text { for } \psi \in C[0,1], t \geq 0 \text { and } x \in[0,1],
$$

where

$$
K(t, x)=\exp \left\{-\int_{0}^{t}\left(\delta(\chi(-s, x))+V^{\prime}(\chi(-s, x))\right) d s\right\} .
$$

Proof. The proof is similar to the proof of Proposition 2.4 in 10].

We denote by $C\left(\Omega_{\Theta}\right)$ the space of continuous function on $\Omega_{\Theta}$, endowed with the norm $\|\cdot\|_{\Omega_{\Theta}}$, defined by

$$
\|\Upsilon\|_{\Omega_{\Theta}}:=\sup _{(m, a) \in \Omega_{\Theta}}|\Upsilon(m, a)|, \quad \text { for } \Upsilon \in C\left(\Omega_{\Theta}\right) .
$$

Now, we can consider an integrated formulation of Problem (16)-(19), given by the variation of constant formula associated to the $C_{0}$-semigroup $(T(t))_{t \geq 0}$. That is the following definition.

Definition 3.1. Let $\Gamma \in C\left(\Omega_{\Theta}\right)$ and $\mu$ be a function such that $\bar{\mu} \in C[0,1]$, with $\bar{\mu}$ given by (14). An integrated solution of Problem (10)-(19) is a continuous solution of the system

$$
\begin{aligned}
& N(t, m)=K(t, m) \bar{\mu}(\chi(-t, m)) \\
& -\int_{0}^{t} K(t-s, m) \beta(\chi(-(t-s), m), N(s, \chi(-(t-s), m))) N(s, \chi(-(t-s), m)) d s \\
& +\int_{0}^{t} K(t-s, m) F(s, \chi(-(t-s), m), N(s-\tau(\Delta(\chi(-(t-s), m))), \Delta(\chi(-(t-s), m)))) d s,
\end{aligned}
$$

and

$$
\begin{aligned}
& P(t, m)=\xi(t, m) \bar{\Gamma}(\chi(-t, m)) \\
& +\int_{0}^{t} \xi(t-s, m) \beta(\chi(-(t-s), m), N(s, \chi(-(t-s), m))) N(s, \chi(-(t-s), m)) d s \\
& -\int_{0}^{t} \xi(t-s, m) G(s, \chi(-(t-s), m), N(s-\tau(\Theta(\chi(-(t-s), m))), \Theta(\chi(-(t-s), m)))) d s,
\end{aligned}
$$

for $t \geq 0$ and $m \in[0,1]$, where $F$ and $G$ are given by (29) and (23) and $\bar{\Gamma}$ is given by (18).

The extension given by (24) allows the second integrals, in the expressions (26) and (27), to be well defined.

In order to obtain a result of local existence for the solutions of System (26)-(27), we first focus on Equation (26). We show, in the next theorem, that Equation (26) has a unique local solution, which depends continuously on the initial conditions. 
Theorem 3.1. Assume that the mapping $x \mapsto x \beta(m, x)$ is locally Lipschitz continuous for all $m \in[0,1]$, that is, for all $r>0$, there exists $L(r) \geq 0$ such that

$$
|x \beta(m, x)-y \beta(m, y)| \leq L(r)|x-y|, \quad \text { if }|x|<r,|y|<r \text { and } m \in[0,1] .
$$

If $\Gamma \in C\left(\Omega_{\Theta}\right)$ and $\mu$ is a function such that $\bar{\mu} \in C[0,1]$, then, there exists $T_{\text {max }}>0$ such that Equation 20) has a unique continuous solution $N^{\bar{\mu}, \Gamma}$ defined on a maximal domain $\left[0, T_{\max }\right) \times[0,1]$, and either

$$
T_{\max }=+\infty \quad \text { or } \quad \limsup _{t \rightarrow T_{\max }^{-}}\left\|N^{\bar{\mu}, \Gamma}(t, .)\right\|=+\infty
$$

Furthermore, $N^{\bar{\mu}, \Gamma}(t,$.$) is a continuous function of \bar{\mu}$ and $\Gamma$, in the sense that, if $t \in\left(0, T_{\max }\right), \bar{\mu}_{1} \in C[0,1]$ and $\Gamma_{1} \in C\left(\Omega_{\Theta}\right)$, then there exist a continuous positive function $C:[0,+\infty) \rightarrow \mathbb{R}$ and a constant $\varepsilon>0$ such that, for $\bar{\mu}_{2} \in C[0,1]$ and $\Gamma_{2} \in C\left(\Omega_{\Theta}\right)$ such that $N^{\bar{\mu}_{2}, \Gamma_{2}}$ is defined on $[0, t] \times[0,1]$ and

$$
\left\|\bar{\mu}_{1}-\bar{\mu}_{2}\right\|<\varepsilon \quad \text { and } \quad\left\|\Gamma_{1}-\Gamma_{2}\right\|_{\Omega_{\Theta}}<\varepsilon,
$$

we get

$$
\left\|N^{\bar{\mu}_{1}, \Gamma_{1}}(s, .)-N^{\bar{\mu}_{2}, \Gamma_{2}}(s, .)\right\| \leq C(t)\left(\left\|\bar{\mu}_{1}-\bar{\mu}_{2}\right\|+\left\|\Gamma_{1}-\Gamma_{2}\right\|_{\Omega_{\Theta}}\right), \quad \text { for } s \in[0, t] .
$$

Proof. We put

$$
r=\|\bar{\mu}\|+1 \text {. }
$$

Let $T>0$ be fixed. We consider the following set,

$$
X(\bar{\mu})=\left\{N \in C([0, T] \times[0,1]) ; N(0, .)=\bar{\mu} \text { on }[0,1] \text { and } \sup _{(t, m) \in[0, T] \times[0,1]}|N(t, m)-\bar{\mu}(m)| \leq 1\right\}
$$

where $C([0, T] \times[0,1])$ is endowed with the uniform norm. $X(\bar{\mu})$ is a non-empty closed convex subset of $C([0, T] \times[0,1])$.

We define the operator $H: C([0, T] \times[0,1]) \rightarrow C([0, T] \times[0,1])$ by

$$
\begin{aligned}
& H(N)(t, m)=K(t, m) \bar{\mu}(\chi(-t, m)) \\
& -\int_{0}^{t} K(t-s, m) \beta(\chi(-(t-s), m), N(s, \chi(-(t-s), m))) N(s, \chi(-(t-s), m)) d s \\
& +\int_{0}^{t} K(t-s, m) F(s, \chi(-(t-s), m), N(s-\tau(\Delta(\chi(-(t-s), m))), \Delta(\chi(-(t-s), m)))) d s .
\end{aligned}
$$

$H$ is continuous in $C([0, T] \times[0,1])$. Our objective is to show that $H$ is a contraction from $X(\bar{\mu})$ into itself.

Let $N \in X(\bar{\mu})$. It is clear that $H(N)(0,)=.\bar{\mu}$. On the other hand, we have, for $(t, m) \in[0, T] \times[0,1]$,

$$
\begin{aligned}
& |H(N)(t, m)-\bar{\mu}(m)| \leq|K(t, m) \bar{\mu}(\chi(-t, m))-\bar{\mu}(m)| \\
& +\left|\int_{0}^{t} K(t-s, m) \beta(\chi(-(t-s), m), N(s, \chi(-(t-s), m))) N(s, \chi(-(t-s), m)) d s\right| \\
& +\left|\int_{0}^{t} K(t-s, m) F(s, \chi(-(t-s), m), N(s-\tau(\Delta(\chi(-(t-s), m))), \Delta(\chi(-(t-s), m)))) d s\right| .
\end{aligned}
$$


Since $K$ is continuous on $[0, T] \times[0,1]$, then there exists $\widetilde{K} \geq 0$ such that

$$
|K(t, m)| \leq \widetilde{K}, \quad \text { for }(t, m) \in[0, T] \times[0,1] .
$$

Since $N \in X(\bar{\mu})$, then

$$
|N(t, m)| \leq 1+\|\bar{\mu}\|=r .
$$

This implies that there exists $M:=\max \{\widetilde{M},\|\zeta\| r L(r)\} \geq 0$ such that, for $(t, m) \in[0, T] \times[0,1]$ and $s \in[0, t]$,

$$
|F(s, \chi(-(t-s), m), N(s-\tau(\Delta(\chi(-(t-s), m))), \Delta(\chi(-(t-s), m))))| \leq M,
$$

where

$$
\widetilde{M}:=\sup _{(m, t) \in \Omega_{\Delta}}\left|2\left(g^{-1}\right)^{\prime}(m) \xi\left(t, g^{-1}(m)\right) \Gamma\left(\chi\left(-t, g^{-1}(m)\right), \tau(\Delta(m))-t\right)\right|,
$$

and $\zeta$ is given by (20). Hence, we obtain that

$$
|H(N)(t, m)-\bar{\mu}(m)| \leq|K(t, m) \bar{\mu}(\chi(-t, m))-\bar{\mu}(m)|+\widetilde{K}(r L(r)+M) t .
$$

Let us recall that $K(0, m)=1, \chi(0, m)=m$ and $(t, m) \mapsto K(t, m) \bar{\mu}(\chi(-t, m))$ is continuous. Then, we can choose $T>0$ such that

$$
\sup _{(t, m) \in[0, T] \times[0,1]}\{|K(t, m) \bar{\mu}(\chi(-t, m))-\bar{\mu}(m)|+\widetilde{K}(r L(r)+M) t\}<1 .
$$

Consequently,

$$
|H(N)(t, m)-\bar{\mu}(m)| \leq 1, \quad \text { for }(t, m) \in[0, T] \times[0,1]
$$

and $H(X(\bar{\mu})) \subset X(\bar{\mu})$.

Now, we show that $H$ is a contraction on $X(\bar{\mu})$.

Let $N_{1} \in X(\bar{\mu})$ and $N_{2} \in X(\bar{\mu})$. Then,

$$
\begin{aligned}
& \left|H\left(N_{1}\right)(t, m)-H\left(N_{2}\right)(t, m)\right| \\
& \leq \mid \int_{0}^{t} K(t-s, m)\left[\beta\left(\chi(-(t-s), m), N_{1}(s, \chi(-(t-s), m))\right) N_{1}(s, \chi(-(t-s), m))\right. \\
& \left.-\beta\left(\chi(-(t-s), m), N_{2}(s, \chi(-(t-s), m))\right) N_{2}(s, \chi(-(t-s), m))\right] d s \mid \\
& +\mid \int_{0}^{t} K(t-s, m)\left[F\left(s, \chi(-(t-s), m), N_{1}(s-\tau(\Delta(\chi(-(t-s), m))), \Delta(\chi(-(t-s), m)))\right)\right. \\
& \left.-F\left(s, \chi(-(t-s), m), N_{2}(s-\tau(\Delta(\chi(-(t-s), m))), \Delta(\chi(-(t-s), m)))\right)\right] d s \mid, \\
& \leq \widetilde{K}(1+\|\zeta\|) L(r) T \sup _{(t, m) \in[0, T] \times[0,1]}\left|N_{1}(t, m)-N_{2}(t, m)\right|
\end{aligned}
$$

Since $r \geq 1$ and $\|\zeta\| r L(r) \leq M$, then Condition (28) implies that

$$
\widetilde{K}(1+\|\zeta\|) L(r) T \leq \widetilde{K}(1+\|\zeta\|) r L(r) T \leq \widetilde{K}(r L(r)+M) T<1 .
$$


Hence, $H$ is a contraction from $X(\bar{\mu})$ into itself. Therefore, there exists a unique $N \in X(\bar{\mu})$ such that $N$ satisfies Equation (26) for $(t, m) \in[0, T] \times[0,1]$.

Let $N$ be the maximal solution of Equation (26), defined on its maximal domain $\left[0, T_{\max }\right) \times[0,1]$.

Assume that

$$
T_{\max }<+\infty \quad \text { and } \quad \limsup _{t \rightarrow T_{\max }^{-}}\|N(t, .)\|<+\infty .
$$

Then, there exists $r>0$ such that

$$
\|N(t, .)\|<r, \quad \text { for } t \in\left[0, T_{\max }\right) .
$$

Let $(t, m) \in\left[0, T_{\max }\right) \times[0,1]$ and $h>0$ such that $t+h \in\left[0, T_{\max }\right)$. Then,

$$
\begin{aligned}
& |N(t+h, m)-N(t, m)| \\
& \leq|K(t+h, m) \bar{\mu}(\chi(-(t+h), m))-K(t, m) \bar{\mu}(\chi(-t, m))| \\
& +\mid \int_{0}^{t+h} K(t+h-s, m) \beta(\chi(-(t+h-s), m), N(s, \chi(-(t+h-s), m))) N(s, \chi(-(t+h-s), m)) d s \\
& +\mid \int_{0}^{t+h} K(t+h-s, m) \times \\
& \quad-\int_{0}^{t} K(t-s, m) \beta(\chi(-(t-s), m), N(s, \chi(-(t-s), m))) N(s, \chi(-(t-s), m)) d s \mid \\
& \quad F(s, \chi(-(t+h-s), m), N(s-\tau(\Delta(\chi(-(t+h-s), m))), \Delta(\chi(-(t+h-s), m)))) d s \\
& \quad-\int_{0}^{t} K(t-s, m) F(s, \chi(-(t-s), m), N(s-\tau(\Delta(\chi(-(t-s), m))), \Delta(\chi(-(t-s), m)))) d s \mid \\
& \leq|K(t+h, m) \bar{\mu}(\chi(-(t+h), m))-K(t, m) \bar{\mu}(\chi(-t, m))|+\left|I_{1}\right|+\left|I_{2}\right| .
\end{aligned}
$$


Since

$$
\begin{aligned}
& I_{1}:=\int_{0}^{t+h} K(t+h-s, m) \beta(\chi(-(t+h-s), m), N(s, \chi(-(t+h-s), m))) N(s, \chi(-(t+h-s), m)) d s \\
& -\int_{0}^{t} K(t-s, m) \beta(\chi(-(t-s), m), N(s, \chi(-(t-s), m))) N(s, \chi(-(t-s), m)) d s \\
& =\int_{-h}^{t} K(t-s, m) \beta(\chi(-(t-s), m), N(s+h, \chi(-(t-s), m))) N(s+h, \chi(-(t-s), m)) d s \\
& -\int_{0}^{t} K(t-s, m) \beta(\chi(-(t-s), m), N(s, \chi(-(t-s), m))) N(s, \chi(-(t-s), m)) d s \\
& =\int_{-h}^{0} K(t-s, m) \beta(\chi(-(t-s), m), N(s+h, \chi(-(t-s), m))) N(s+h, \chi(-(t-s), m)) d s \\
& +\int_{0}^{t} K(t-s, m)[\beta(\chi(-(t-s), m), N(s+h, \chi(-(t-s), m))) N(s+h, \chi(-(t-s), m)) \\
& \quad-\beta(\chi(-(t-s), m), N(s, \chi(-(t-s), m))) N(s, \chi(-(t-s), m))] d s
\end{aligned}
$$

then

$$
\left|I_{1}\right| \leq \widetilde{K} r L(r) h+\widetilde{K} L(r) \int_{0}^{t}|N(s+h, \chi(-(t-s), m))-N(s, \chi(-(t-s), m))| d s .
$$

By using the same reasonning, we also obtain that

$$
\begin{array}{r}
\left|I_{2}\right| \leq \widetilde{K} M h+\varrho_{1}(h)+\widetilde{K}\|\zeta\| L(r) \int_{0}^{t} \mid N(s+h-\tau(\Delta(\chi(-(t-s), m))), \Delta(\chi(-(t-s), m))) \\
-N(s-\tau(\Delta(\chi(-(t-s), m))), \Delta(\chi(-(t-s), m))) \mid d s,
\end{array}
$$

with $\varrho_{1}(h)$ independent of $(t, m)$ and such that $\lim _{h \rightarrow 0} \varrho_{1}(h)=0$.

One can remark that, if $t_{1}>0, t_{2}>0$ and $m \in[0,1]$, then

$$
K\left(t_{1}+t_{2}, m\right)=K\left(t_{1}, m\right) K\left(t_{2}, \chi\left(-t_{1}, m\right)\right)=K\left(t_{2}, m\right) K\left(t_{1}, \chi\left(-t_{2}, m\right)\right) .
$$

This yields to

$$
\begin{aligned}
& |N(t+h, m)-N(t, m)| \\
& \leq \widetilde{K}\left(\varrho_{2}(h)+r L(r) h+M h\right)+\varrho_{1}(h)+\widetilde{K} L(r) \int_{0}^{t}\|N(s+h, .)-N(s, .)\| d s \\
& +\widetilde{K}\|\zeta\| L(r) \int_{0}^{t}\|N(s+h-\tau(\Delta(\chi(-(t-s), m))), .)-N(s-\tau(\Delta(\chi(-(t-s), m))), .)\| d s,
\end{aligned}
$$

where

$$
\varrho_{2}(h)=\sup _{(t, m) \in\left[0, T_{\max }\right) \times[0,1]}|K(h, \chi(-t, m)) \bar{\mu}(\chi(-(t+h), m))-\bar{\mu}(\chi(-t, m))| \underset{h \rightarrow 0}{\longrightarrow} 0 .
$$


We set

$$
\varrho(h)=\widetilde{K}\left(\varrho_{2}(h)+r L(r) h+M h\right)+\varrho_{1}(h) .
$$

Hence, for $(t, m) \in\left[0, T_{\max }\right) \times[0,1], h>0$ and $\theta \in\left[-\tau_{\max }, 0\right]$ such that $t+h \in\left[0, T_{\max }\right)$ and $t+\theta \geq 0$,

$$
\begin{aligned}
& |N(t+h+\theta, m)-N(t+\theta, m)| \leq \varrho(h)+\widetilde{K} L(r) \int_{0}^{t+\theta}\|N(s+h, .)-N(s, .)\| d s \\
& +\widetilde{K}\|\zeta\| L(r) \int_{0}^{t+\theta}\|N(s+h-\tau(\Delta(\chi(-(t-s), m))), .)-N(s-\tau(\Delta(\chi(-(t-s), m))), .)\| d s .
\end{aligned}
$$

On the other hand,

$$
N(t, m)=\bar{\mu}(m), \quad \text { for } t \in\left[-\tau_{\max }, 0\right] \text { and } m \in[0,1]
$$

This implies that

$\sup _{\theta \in\left[-\tau_{\max }, 0\right]}\|N(t+h+\theta,)-.N(t+\theta,).\| \leq \varrho(h)+\widetilde{K}(\|\zeta\|+1) L(r) \int_{0}^{t} \sup _{\theta \in\left[-\tau_{\max }, 0\right]}\|N(s+h+\theta,)-.N(s+\theta,)\| d s$.

By using the Gronwall's inequality, it follows that

$$
\sup _{\theta \in\left[-\tau_{\max }, 0\right]}\|N(t+h+\theta, .)-N(t+\theta, .)\| \leq \varrho(h) e^{\widetilde{K}(\|\zeta\|+1) L(r) T_{\max }} .
$$

Hence,

$$
\|N(t+h, .)-N(t, .)\| \leq \varrho(h) e^{\widetilde{K}(\|\zeta\|+1) L(r) T_{\max }}
$$

with

$$
\lim _{h \rightarrow 0} \varrho(h)=0 .
$$

Using the same reasonning, we can show a same result for $h<0$.

It follows immediatly that

$$
\lim _{t \rightarrow T_{\max }^{-}} N(t, .) \text { exists. }
$$

This implies that $N$ can be extended continuously to $T_{\max }$, which contradicts the maximality of $\left[0, T_{\max }\right)$. Hence,

$$
T_{\max }=+\infty \quad \text { or } \quad \limsup _{t \rightarrow T^{-}}\|N(t, .)\|=+\infty .
$$

Now, we denote by $N^{\bar{\mu}_{1}, \Gamma_{1}}$ the solution of Equation (26) for the initial data $\bar{\mu}_{1}$ and $\Gamma_{1}$, defined on its maximal domain $\left[0, T_{\max }\right) \times[0,1]$. Let $t \in\left(0, T_{\max }\right)$ be fixed. We put

$$
r(t)=\sup _{s \in[0, t]}\left\|N^{\bar{\mu}_{1}, \Gamma_{1}}(s, .)\right\| \quad \text { and } \quad R(t)=1+r(t) .
$$

We set

$$
\widetilde{C}:=\sup _{(m, t) \in \Omega_{\Theta}}\left|2\left(g^{-1}\right)^{\prime}(m) \xi\left(t, g^{-1}(m)\right)\right|,
$$

and

$$
C(t)=\widetilde{K} \max \{1, \widetilde{C}\} e^{L(R(t)) \widetilde{K}(1+\|\zeta\|) t} .
$$

Let $0<\varepsilon<1$ be such that $2 C(t) \varepsilon \in(0,1)$ and let $\bar{\mu}_{2} \in C[0,1]$ and $\Gamma_{2} \in C\left(\Omega_{\Theta}\right)$ such that $N^{\bar{\mu}_{2}, \Gamma_{2}}$ is defined on $[0, t] \times[0,1]$ and

$$
\left\|\bar{\mu}_{1}-\bar{\mu}_{2}\right\|<\varepsilon \quad \text { and } \quad\left\|\Gamma_{1}-\Gamma_{2}\right\|_{\Omega_{\Theta}}<\varepsilon \text {. }
$$


Then, there exists $s>0$ such that $\left\|N^{\bar{\mu}_{2}, \Gamma_{2}}(\sigma,).\right\| \leq R(t)$ for all $\sigma \in[0, s]$. Let

$$
t_{0}=\sup \left\{s>0 ;\left\|N^{\bar{\mu}_{2}, \Gamma_{2}}(\sigma, .)\right\| \leq R(t), \text { for } \sigma \in[0, s]\right\} \text {. }
$$

If we suppose that $t_{0}<t$, then $N^{\bar{\mu}_{2}, \Gamma_{2}}$ is defined on $\left[0, t_{0}\right] \times[0,1]$ and satisfies, for $s \in\left[0, t_{0}\right]$ and $m \in[0,1]$,

$$
\begin{aligned}
\left|N^{\bar{\mu}_{1}, \Gamma_{1}}(s, m)-N^{\bar{\mu}_{2}, \Gamma_{2}}(s, m)\right| & \leq \widetilde{K}\left(\left\|\bar{\mu}_{1}-\bar{\mu}_{2}\right\|+\widetilde{C}\left\|\Gamma_{1}-\Gamma_{2}\right\|_{\Omega_{\Theta}}\right) \\
& +\widetilde{K}(1+\|\zeta\|) L(R(t)) \int_{0}^{s}\left\|N^{\bar{\mu}_{1}, \Gamma_{1}}(\sigma, .)-N^{\bar{\mu}_{2}, \Gamma_{2}}(\sigma, .)\right\| d \sigma .
\end{aligned}
$$

Therefore, using the Gronwall's Inequality, we obtain

$$
\left|N^{\bar{\mu}_{1}, \Gamma_{1}}(s, m)-N^{\bar{\mu}_{2}, \Gamma_{2}}(s, m)\right| \leq C(t)\left(\left\|\bar{\mu}_{1}-\bar{\mu}_{2}\right\|+\left\|\Gamma_{1}-\Gamma_{2}\right\|_{\Omega_{\Theta}}\right), \quad \text { for } s \in\left[0, t_{0}\right] .
$$

This implies, in particular, that

$$
\left\|N^{\bar{\mu}_{2}, \Gamma_{2}}(s, .)\right\| \leq 2 C(t) \varepsilon+\left\|N^{\bar{\mu}_{1}, \Gamma_{1}}(s, .)\right\|<1+r(t)=R(t), \quad \text { for } s \in\left[0, t_{0}\right] .
$$

This contradicts the definition of $t_{0}$. Hence, $t_{0} \geq t$.

That means that (29) is satisfied for each $t \in\left[0, T_{\max }\right)$. Then, we deduce the continuous dependence of the solution with the initial data and the proof is complete.

One can remark that Condition $(15)$ is not needed to prove Theorem 3.1.

By using Theorem 3.1, we can deduce the following result, which deals with the existence of solutions of Problem (26)- 27).

Corollary 3.1. Under the assumptions of Theorem 3.1, System (20)-(27) has a unique continuous maximal solution $(N, P)$, defined on $\left[0, T_{\max }\right) \times[0,1]$.

Proof. Under the assumptions of Theorem 3.1, Equation (26) has a unique continuous maximal solution $N$ defined on $\left[0, T_{\max }\right) \times[0,1]$. Then, we easily obtain the existence and uniqueness of a solution of Equation (27) on $\left[0, T_{\max }\right) \times[0,1]$.

We can use the results of local existence, given by Theorem 3.1 and Corollary 3.1, to investigate the global existence of the solutions of System (26)-(27). This is done in the next theorem.

Theorem 3.2. Under the assumptions of Theorem 3.1 and the assumption that the mapping $x \mapsto \beta(m, x)$ is uniformly bounded, the unique solution of System (20)-(27) is global, that means, it is defined for all $t \geq 0$.

Proof. We assume that there exists $\widetilde{\beta} \geq 0$ such that

$$
|\beta(m, x)| \leq \widetilde{\beta}, \quad \text { for all } m \in[0,1] \text { and } x \in \mathbb{R} .
$$

Then, for $t \in\left[0, T_{\max }\right)$ and $m \in[0,1]$, we get

$$
\begin{aligned}
|N(t, m)| & \leq \widetilde{K}\|\bar{\mu}\|+\widetilde{K} \widetilde{\beta} \int_{0}^{t}\|N(s, .)\| d s \\
& +\widetilde{K} \widetilde{M} t+\widetilde{K} \widetilde{\beta}\|\zeta\| \int_{0}^{t}\|N(s-\tau(\Delta(\chi(-(t-s), m))), .)\| d s .
\end{aligned}
$$


Let $\theta \in\left[-\tau_{\max }, 0\right]$ be such that $t+\theta \geq 0$. Then,

$$
|N(t+\theta, m)| \leq \widetilde{K}(\|\bar{\mu}\|+\widetilde{M} t)+\widetilde{K} \widetilde{\beta}(\|\zeta\|+1) \int_{0}^{t} \sup _{\bar{\theta} \in\left[-\tau_{\max }, 0\right]}\|N(s+\bar{\theta}, .)\| d s .
$$

On the other hand, if $-\tau_{\max } \leq t+\theta \leq 0$, then

$$
|N(t+\theta, m)| \leq \widetilde{K}\|\bar{\mu}\|
$$

because $\widetilde{K} \geq 1$. Hence,

$$
\sup _{\theta \in\left[-\tau_{\max }, 0\right]}\|N(t+\theta, .)\| \leq \widetilde{K}(\|\bar{\mu}\|+\widetilde{M} t)+\widetilde{K} \widetilde{\beta}(\|\zeta\|+1) \int_{0}^{t} \sup _{\theta \in\left[-\tau_{\max }, 0\right]}\|N(s+\theta, .)\| d s .
$$

By using the Gronwall's inequality, we deduce that

$$
\|N(t, .)\| \leq \sup _{\theta \in\left[-\tau_{\max }, 0\right]}\|N(t+\theta, .)\| \leq \widetilde{K}(\|\bar{\mu}\|+\widetilde{M} t) e^{\widetilde{K} \widetilde{\beta}(\|\zeta\|+1) t}:=f(t) .
$$

Since $f$ is continuous on $\left[0, T_{\max }\right]$, then

$$
\limsup _{t \rightarrow T_{\max }^{-}}\|N(t, .)\|<+\infty .
$$

We deduce that $T_{\max }=+\infty$ and the solution of (26) is global.

Finally, we easily obtain that the unique maximal solution of Equation (27) is also global.

Corollary 3.2. Assume that the mapping $x \mapsto x \beta(m, x)$ is Lipschitz continuous for all $m \in[0,1]$. Then, for $\Gamma \in C\left(\Omega_{\Theta}\right)$ and $\mu$ such that $\bar{\mu} \in C[0,1]$, the unique solution of System (20)-(27) is global.

We have studied, throughout Theorems 3.1 and 3.2, the local and global existence of the solutions of (26) and of Problem (26)-(27). Before we investigate the positivity of these solutions in the next section, we can ask for regularity results for these solutions. This is presented in the following remark.

Remark 1. Under classical assumptions on the function $\beta$ and the initial data $\Gamma$ and $\mu$, we can obtain regularity results for the solution of System (20)-(27). This may be done by using the same idea as in the work of Travis and Webb [23].

\section{Positivity of solutions}

Since we study a biological population, it is necessary to obtain the positivity of the solutions of System (26)-(27) to ensure that the model is well-posed. First, we focus our study on the solutions of Equation (26). We use a method given by Webb 24 in 1985 and developped by Kato [14 to obtain the positivity of the solutions of Equation (26).

Let $\Gamma \in C\left(\Omega_{\Theta}\right)$ and $\mu$ be a function such that $\bar{\mu} \in C[0,1], \bar{\mu}$ given by (14). Let $T>0$ be fixed. We consider the family of operators $H^{a}: C([0, T) \times[0,1]) \rightarrow C([0, T) \times[0,1])$ defined, for $a \in C[0,1]$, 
$(t, m) \in[0, T) \times[0,1]$ and $N \in C([0, T) \times[0,1])$, by

$$
\begin{aligned}
& H^{a}(N)(t, m)=e^{-t a(\chi(-t, m))} K(t, m) \bar{\mu}(\chi(-t, m)) \\
& +\int_{0}^{t} e^{-(t-s) a(\chi(-t, m))} K(t-s, m)[a(\chi(-(t-s), m)) N(s, \chi(-(t-s), m)) \\
& -\beta(\chi(-(t-s), m), N(s, \chi(-(t-s), m))) N(s, \chi(-(t-s), m)) \\
& +F(s, \chi(-(t-s), m), N(s-\tau(\Delta(\chi(-(t-s), m))), \Delta(\chi(-(t-s), m))))] d s .
\end{aligned}
$$

Let $a \in C[0,1],(t, m) \in(0, T) \times[0,1]$ and $N \in C([0, T) \times[0,1])$ be fixed. We consider the mapping $w^{a}:[0, t] \rightarrow \mathbb{R}$ defined, for $s \in[0, t]$, by

$$
w^{a}(s)=H^{a}(N)(s, \chi(-(t-s), m)) .
$$

Then, we can prove the following lemmas.

Lemma 4.1. The function $w^{a}$ is differentiable on $[0, t]$ and satisfies

$$
\left\{\begin{aligned}
\frac{d}{d s} w^{a}(s) & =-\nu^{a}(s) w^{a}(s)+f^{a}(s), \quad \text { for } s \in[0, t] \\
w^{a}(0) & =\bar{\mu}(\chi(-t, m))
\end{aligned}\right.
$$

with

$$
\begin{aligned}
f^{a}(s) & =a(\chi(-(t-s), m)) N(s, \chi(-(t-s), m)) \\
& -N(s, \chi(-(t-s), m)) \beta(\chi(-(t-s), m), N(s, \chi(-(t-s), m))) \\
& +F(s, \chi(-(t-s), m), N(s-\tau(\Delta(\chi(-(t-s), m))), \Delta(\chi(-(t-s), m)))),
\end{aligned}
$$

and

$$
\nu^{a}(s)=a(\chi(-t, m))+\delta(\chi(-(t-s), m))+V^{\prime}(\chi(-(t-s), m)) .
$$

Proof. First, one can remark that, if $t_{1}$ and $t_{2}$ are positive, then

$$
\chi\left(-\left(t_{1}+t_{2}\right), m\right)=\chi\left(-t_{1}, \chi\left(-t_{2}, m\right)\right) .
$$

It follows that, for $s \in[0, t]$ and $h \neq 0$ such that $s+h \in[0, t]$, we have

$$
\frac{1}{h}\left(w^{a}(s+h)-w^{a}(s)\right)=W_{1, h}+W_{2, h},
$$

with

$$
W_{1, h}=\frac{1}{h}\left[e^{-h a(\chi(-t, m))} K(h, \chi(-(t-s-h), m))-1\right] e^{-s a(\chi(-t, m))} K(s, \chi(-(t-s), m)) \bar{\mu}(\chi(-t, m)),
$$


and

$$
\begin{aligned}
W_{2, h}= & \frac{1}{h} \int_{0}^{s+h} e^{-(s+h-\sigma) a(\chi(-t, m))} K(s+h-\sigma, \chi(-(t-s-h), m)) \times \\
& {[a(\chi(-(t-\sigma), m)) N(\sigma, \chi(-(t-\sigma), m))} \\
& -N(\sigma, \chi(-(t-\sigma), m)) \beta(\chi(-(t-\sigma), m), N(\sigma, \chi(-(t-\sigma), m))) \\
& +F(\sigma, \chi(-(t-\sigma), m), N(\sigma-\tau(\Delta(\chi(-(t-\sigma), m))), \Delta(\chi(-(t-\sigma), m))))] d \sigma \\
- & \frac{1}{h} \int_{0}^{s} e^{-(s-\sigma) a(\chi(-t, m))} K(s-\sigma, \chi(-(t-s), m)) \times \\
& {[a(\chi(-(t-\sigma), m)) N(\sigma, \chi(-(t-\sigma), m))} \\
& -N(\sigma, \chi(-(t-\sigma), m)) \beta(\chi(-(t-\sigma), m), N(\sigma, \chi(-(t-\sigma), m))) \\
& +F(\sigma, \chi(-(t-\sigma), m), N(\sigma-\tau(\Delta(\chi(-(t-\sigma), m))), \Delta(\chi(-(t-\sigma), m))))] d \sigma .
\end{aligned}
$$

Hence, we easily obtain that

$$
\lim _{h \rightarrow 0} W_{1, h}=-\nu^{a}(s) e^{-s a(\chi(-t, m))} K(s, \chi(-(t-s), m)) \bar{\mu}(\chi(-t, m)) .
$$


By the same way, we have

$$
\begin{aligned}
& W_{2, h}=\frac{1}{h}\left[e^{-h a(\chi(-t, m))} K(h, \chi(-(t-s-h), m))-1\right] \times \\
& \int_{0}^{s} e^{-(s-\sigma) a(\chi(-t, m))} K(s-\sigma, \chi(-(t-s), m))[a(\chi(-(t-\sigma), m)) N(\sigma, \chi(-(t-\sigma), m)) \\
& -N(\sigma, \chi(-(t-\sigma), m)) \beta(\chi(-(t-\sigma), m), N(\sigma, \chi(-(t-\sigma), m))) \\
& +F(\sigma, \chi(-(t-\sigma), m), N(\sigma-\tau(\Delta(\chi(-(t-\sigma), m))), \Delta(\chi(-(t-\sigma), m))))] d \sigma \\
& +\frac{1}{h} e^{-h a(\chi(-t, m))} K(h, \chi(-(t-s-h), m)) \int_{s}^{s+h} e^{-(s-\sigma) a(\chi(-t, m))} K(s-\sigma, \chi(-(t-s), m)) \times \\
& {[a(\chi(-(t-\sigma), m)) N(\sigma, \chi(-(t-\sigma), m))} \\
& -N(\sigma, \chi(-(t-\sigma), m)) \beta(\chi(-(t-\sigma), m), N(\sigma, \chi(-(t-\sigma), m))) \\
& +F(\sigma, \chi(-(t-\sigma), m), N(\sigma-\tau(\Delta(\chi(-(t-\sigma), m))), \Delta(\chi(-(t-\sigma), m))))] d \sigma .
\end{aligned}
$$

Therefore,

$$
\begin{aligned}
\lim _{h \rightarrow 0} W_{2, h}= & -\nu^{a}(s) \int_{0}^{s} e^{-(s-\sigma) a(\chi(-t, m))} K(s-\sigma, \chi(-(t-s), m)) \times \\
& {[a(\chi(-(t-\sigma), m)) N(\sigma, \chi(-(t-\sigma), m))} \\
& -N(\sigma, \chi(-(t-\sigma), m)) \beta(\chi(-(t-\sigma), m), N(\sigma, \chi(-(t-\sigma), m))) \\
& +F(\sigma, \chi(-(t-\sigma), m), N(\sigma-\tau(\Delta(\chi(-(t-\sigma), m))), \Delta(\chi(-(t-\sigma), m))))] d \sigma \\
& +a(\chi(-(t-s), m)) N(s, \chi(-(t-s), m)) \\
& -N(s, \chi(-(t-s), m)) \beta(\chi(-(t-s), m), N(s, \chi(-(t-s), m))) \\
& +F(s, \chi(-(t-s), m), N(s-\tau(\Delta(\chi(-(t-s), m))), \Delta(\chi(-(t-s), m)))) .
\end{aligned}
$$


Hence, $\lim _{h \rightarrow 0} \frac{1}{h}\left(w^{a}(s+h)-w^{a}(s)\right)$ exists and is equal to $-\nu^{a}(s) w^{a}(s)+f^{a}(s)$.

We obtain Equation (31) and the proof of the lemma is complete.

Lemma 4.2. Let $a, b \in C[0,1]$. Then,

$$
\begin{aligned}
H^{b}(N)(t, m)= & H^{a}(N)(t, m)+\int_{0}^{t} e^{-(t-s) b(\chi(-t, m))} K(t-s, m) \times \\
& (b(\chi(-(t-s), m))-a(\chi(-(t-s), m)))\left[N(s, \chi(-(t-s), m))-w^{a}(s)\right] d s .
\end{aligned}
$$

Proof. From Equation (31), it follows that

$$
\begin{aligned}
\frac{d}{d s}\left[w^{b}(s)-w^{a}(s)\right]= & -\nu^{b}(s)\left(w^{b}(s)-w^{a}(s)\right) \\
& +(b(\chi(-(t-s), m))-a(\chi(-(t-s), m)))\left[N(s, \chi(-(t-s), m))-w^{a}(s)\right] .
\end{aligned}
$$

Since $w^{b}(0)=w^{a}(0)=\bar{\mu}(\chi(-t, m))$, then

$$
\begin{aligned}
& \left(w^{b}(t)-w^{a}(t)\right) e^{\int_{0}^{t} \nu^{b}(\sigma) d \sigma}= \\
& \int_{0}^{t} e^{\int_{0}^{s} \nu^{b}(\sigma) d \sigma}(b(\chi(-(t-s), m))-a(\chi(-(t-s), m)))\left[N(s, \chi(-(t-s), m))-w^{a}(s)\right] d s .
\end{aligned}
$$

Therefore,

$$
\begin{aligned}
w^{b}(t)-w^{a}(t)= & \int_{0}^{t} e^{-(t-s) b(\chi(-t, m))} K(t-s, m) \times \\
& (b(\chi(-(t-s), m))-a(\chi(-(t-s), m)))\left[N(s, \chi(-(t-s), m))-w^{a}(s)\right] d s .
\end{aligned}
$$

This ends the proof.

Lemma 4.3. Assume that there exist $a \in C[0,1]$ and $N \in C([0, T) \times[0,1])$ such that $H^{a}(N)=N$. Then, for all $b \in C[0,1], H^{b}(N)=N$.

Proof. This is a simple consequence of Lemma 4.2.

Now, we show, under a cellular regulation hypothesis, that the solutions of (26)-(27) are positive. We use the three previous lemmas to show the following theorem.

Theorem 4.1. Assume that the mapping $x \mapsto \beta(m, x)$ satisfies the cellular regulation hypothesis,

$$
(\beta(m, x)-\beta(m, 0)) x \leq 0, \quad \text { for } m \in[0,1] \text { and } x \in \mathbb{R} .
$$

Let $\Gamma \in C\left(\Omega_{\Theta}\right)$ and $\mu$ be a continuous function such that $\bar{\mu} \in C[0,1]$. If $\bar{\mu} \geq 0$ and $\Gamma \geq 0$, then the solutions of Equation (20) are non-negative on their domains.

Proof. Let $N$ be a solution of Equation (26) defined on $[0, T) \times[0,1]$, where $T>0$.

We consider the operator $H^{a}$ defined, for $a \in C[0,1]$, by (30). It is obvious that

$$
H^{0}(N)=N \text {, }
$$

where 0 denotes the null mapping of $C[0,1]$. 
Then, from Lemma 4.3, for all $a \in C[0,1], H^{a}(N)=N$. Hence, if there exists $a \in C[0,1]$ such that $H^{a}(N)(t, m) \geq 0$ for $(t, m) \in[0, T) \times[0,1]$, then we shall obtain that $N(t, m) \geq 0$, for $(t, m) \in[0, T) \times[0,1]$. We set

$$
a(m):=\beta(m, 0), \quad \text { for all } m \in[0,1] .
$$

Then, $a \in C[0,1]$ and Condition (32) implies that

$$
a(m) N(t, m)-N(t, m) \beta(m, N(t, m)) \geq 0, \quad \text { for }(t, m) \in[0, T) \times[0,1] .
$$

Hence,

$$
\begin{aligned}
& \int_{0}^{t} e^{-(t-s) a(\chi(-t, m))} K(t-s, m)[a(\chi(-(t-s), m)) N(s, \chi(-(t-s), m)) \\
& -\beta(\chi(-(t-s), m), N(s, \chi(-(t-s), m))) N(s, \chi(-(t-s), m))] d s
\end{aligned}
$$

is non-negative. We have to show by steps that, for $(t, m) \in[0, T) \times[0,1]$,

$\int_{0}^{t} e^{-(t-s) a(\chi(-t, m))} K(t-s, m) F(s, \chi(-(t-s), m), N(s-\tau(\Delta(\chi(-(t-s), m))), \Delta(\chi(-(t-s), m)))) d s$ is non-negative.

First, it is clear that $H^{a}(N)$ is non-negative on $\Omega_{\Delta}$.

We set

$$
\tau_{\Delta}:=\min _{m \in[0,1]} \tau(\Delta(m))
$$

Since $\tau$ is positive on $[0,1]$, then $\tau_{\Delta}>0$.

Let $m \in[0,1]$. Suppose that $t \in\left[\tau(\Delta(m)), \tau(\Delta(m))+\tau_{\Delta}\right]$. Then,

$$
0 \leq s-\tau(\Delta(\chi(-(t-s), m))) \leq t-\tau_{\Delta} \leq \tau(\Delta(m)), \quad \text { for } s \in[\tau(\Delta(m)), t] .
$$

Since $F \geq 0$ for $(m, t) \in \Omega_{\Delta}$, this yields that

$\int_{0}^{t} e^{-(t-s) a(\chi(-t, m))} K(t-s, m) F(s, \chi(-(t-s), m), N(s-\tau(\Delta(\chi(-(t-s), m))), \Delta(\chi(-(t-s), m)))) d s \geq 0$.

Hence, $H^{a}(N)(t, m) \geq 0$ for $m \in[0,1]$ and $t \in\left[0, \tau(\Delta(m))+\tau_{\Delta}\right]$. This implies that

$$
N(t, m) \geq 0, \quad \text { for } m \in[0,1] \text { and } t \in\left[0, \tau(\Delta(m))+\tau_{\Delta}\right] .
$$

By steps, we show that

$$
N(t, m) \geq 0, \quad \text { for } m \in[0,1] \text { and } t \in\left[0, \tau(\Delta(m))+n \tau_{\Delta}\right] \text { with } n \in \mathbb{N} .
$$

There exists $n \in \mathbb{N}$ such that

$$
\tau(\Delta(m))+n \tau_{\Delta}<T \leq \tau(\Delta(m))+(n+1) \tau_{\Delta} .
$$

Consequently, $N \geq 0$ on its domain $[0, T) \times[0,1]$ and the proof is complete.

We deduce immediatly the following corollary.

Corollary 4.1. Under the assumptions of Theorem 4.1, the solutions of Problem (20)-(27) are nonnegative. 


\section{Local and global stability}

We study, in this section, the local and global stability of the trivial solution of Problem (26)-(27). We first focus on Equation (26).

We set

$$
\widetilde{\delta}:=\inf _{m \in[0,1]}\left(\delta(m)+V^{\prime}(m)\right), \quad \kappa:=\sup _{m \in[0,1]}\left|\left(g^{-1}\right)^{\prime}(m)\right| \quad \text { and } \quad \widetilde{\gamma}:=\inf _{m \in[0,1]}\left(\gamma(m)+V^{\prime}(m)\right) .
$$

We assume that $\widetilde{\gamma} \geq 0$ and that the function $x \mapsto x \beta(m, x)$ satisfies a Lipshitz condition in a neighborhood of zero, that is, there exist $\varepsilon>0$ and $L \geq 0$ such that

$$
|x \beta(m, x)-y \beta(m, y)| \leq L|x-y|, \quad \text { if }|x|<\varepsilon,|y|<\varepsilon \text { and } m \in[0,1] .
$$

Throughout this section, $N^{\bar{\mu}, \Gamma}$ denotes a global solution of Equation (26) associated with the initial data $\mu$ and $\Gamma$.

In order to obtain the local stability of the trivial solution, we first prove a result of invariance for the solutions.

Proposition 5.1. Assume that Condition (39) is satisfied and that

$$
L(1+2 \kappa)<\widetilde{\delta}
$$

Let $\Gamma \in C\left(\Omega_{\Theta}\right)$ and $\mu$ be a continuous function such that $\bar{\mu} \in C[0,1]$. If $\|\bar{\mu}\| \leq \varepsilon$ and $\|\Gamma\|_{\Omega_{\Theta}} \leq \varepsilon L$, where $\varepsilon$ and $L$ are given by (3.), then

$$
\left|N^{\bar{\mu}, \Gamma}(t, m)\right| \leq \varepsilon, \quad \text { for } t \geq 0 \text { and } m \in[0,1]
$$

Proof. We first notice that, under the assumption (34), $\widetilde{\delta}>0$. We consider the sequence defined by

$$
N_{0}(t, m)=K(t, m) \bar{\mu}(\chi(-t, m))
$$

and

$$
N_{n}(t, m)=N_{0}(t, m)-I\left(N_{n-1}\right)(t, m)+J\left(N_{n-1}\right)(t, m),
$$

for $t \geq 0, m \in[0,1]$ and $n \in \mathbb{N}^{*}$, with

$$
I(N)(t, m)=\int_{0}^{t} K(t-s, m) \beta(\chi(-(t-s), m), N(s, \chi(-(t-s), m))) N(s, \chi(-(t-s), m)) d s,
$$

and

$$
J(N)(t, m)=\int_{0}^{t} K(t-s, m) F(s, \chi(-(t-s), m), N(s-\tau(\Delta(\chi(-(t-s), m))), \Delta(\chi(-(t-s), m)))) d s .
$$

Then,

$$
\left|N_{0}(t, m)\right| \leq e^{-\widetilde{\delta} t}\|\bar{\mu}\| \leq e^{-\widetilde{\delta} t} \varepsilon \leq \varepsilon
$$

By induction, we show that

$$
\left|N_{n}(t, m)\right| \leq \varepsilon, \quad \text { for } t \geq 0, m \in[0,1] \text { and } n \in \mathbb{N}^{*}
$$

We assume that $\left|N_{n}(t, m)\right| \leq \varepsilon$.

Let $(t, m) \in[0,+\infty) \times[0,1]$ be fixed. 
One has to notice that, if $0 \leq s \leq \tau(\Delta(\chi(-(t-s), m)))$, then

$$
\begin{aligned}
& \left|F\left(s, \chi(-(t-s), m), N_{n}(s-\tau(\Delta(\chi(-(t-s), m))), \Delta(\chi(-(t-s), m)))\right)\right| \\
& \leq 2 \kappa e^{-\widetilde{\gamma} s}\|\Gamma\|_{\Omega_{\Theta}}, \\
& \leq 2 \kappa \varepsilon L,
\end{aligned}
$$

and, if $s>\tau(\Delta(\chi(-(t-s), m)))$, then

$$
\begin{aligned}
& \left|F\left(s, \chi(-(t-s), m), N_{n}(s-\tau(\Delta(\chi(-(t-s), m))), \Delta(\chi(-(t-s), m)))\right)\right| \\
& \leq\|\zeta\| \mid \beta\left(\Delta(\chi(-(t-s), m)), N_{n}(s-\tau(\Delta(\chi(-(t-s), m))), \Delta(\chi(-(t-s), m)))\right) \times \\
& \quad N_{n}(s-\tau(\Delta(\chi(-(t-s), m))), \Delta(\chi(-(t-s), m))) \mid, \\
& \leq\|\zeta\| \varepsilon L,
\end{aligned}
$$

where $\|\zeta\|$ is given by (25). Since $\|\zeta\| \leq 2 \kappa$, we obtain that

$$
\left|J\left(N_{n}\right)(t, m)\right| \leq 2 \kappa \varepsilon L \int_{0}^{t} e^{-\widetilde{\delta}(t-s)} d s,
$$

and

$$
\begin{aligned}
\left|N_{n+1}(t, m)\right| & \leq\left|N_{0}(t, m)\right|+\left|I\left(N_{n}\right)(t, m)\right|+\left|J\left(N_{n}\right)(t, m)\right|, \\
& \leq \varepsilon e^{-\widetilde{\delta} t}+\varepsilon L(1+2 \kappa) \int_{0}^{t} e^{-\widetilde{\delta}(t-s)} d s, \\
& \leq \varepsilon e^{-\widetilde{\delta} t}+\varepsilon \frac{L}{\widetilde{\delta}}(1+2 \kappa)\left(1-e^{-\widetilde{\delta} t}\right) .
\end{aligned}
$$

By assumption,

$$
\frac{L}{\widetilde{\delta}}(1+2 \kappa)<1
$$

Consequently,

$$
\left|N_{n+1}(t, m)\right| \leq \varepsilon\left(e^{-\widetilde{\delta} t}\left(1-\frac{L}{\widetilde{\delta}}(1+2 \kappa)\right)+\frac{L}{\widetilde{\delta}}(1+2 \kappa)\right) \leq \varepsilon .
$$

We conclude that (35) is true for $n \in \mathbb{N}^{*}$.

By remarking that the sequence $\left(N_{n}\right)_{n \in \mathbb{N}^{*}}$ converges to $N^{\bar{\mu}, \Gamma}$, then we obtain that

$$
\left|N^{\bar{\mu}, \Gamma}(t, m)\right| \leq \varepsilon, \quad \text { for } t \geq 0 \text { and } m \in[0,1],
$$

which ends the proof.

Before we prove the local stability of Equation (26), we rewrite the integrated solution of (26) by using 
a variation of constant formula, for $t \geq \tau_{\max }$ and $m \in[0,1]$. We obtain that

$$
\begin{aligned}
& N(t, m)=K\left(t-\tau_{\max }, m\right) N\left(\tau_{\max }, \chi\left(-\left(t-\tau_{\max }\right), m\right)\right) \\
& -\int_{\tau_{\max }}^{t} K(t-s, m) \beta(\chi(-(t-s), m), N(s, \chi(-(t-s), m))) N(s, \chi(-(t-s), m)) d s \\
& +\int_{\tau_{\max }}^{t} K(t-s, m) \zeta(\chi(-(t-s), m)) N(s-\tau(\Delta(\chi(-(t-s), m))), \Delta(\chi(-(t-s), m))) \times \\
& \beta(\Delta(\chi(-(t-s), m)), N(s-\tau(\Delta(\chi(-(t-s), m))), \Delta(\chi(-(t-s), m)))) d s .
\end{aligned}
$$

Hence, we can show the following theorem, which deals with the local stability of the trivial solution of Equation (26).

Theorem 5.1. Under the assumptions of Proposition 5.1, the trivial solution of Equation (20) is locally exponentially stable, that is, there exist $\bar{t}>0, \epsilon>0, c \geq 0$ and $d \geq 0$ such that, if $\|\bar{\mu}\|<\epsilon$ and $\|\Gamma\|_{\Omega_{\Theta}}<\epsilon$, then

$$
\left\|N^{\bar{\mu}, \Gamma}(t, .)\right\| \leq c e^{-d(t-\bar{t})}, \quad \text { for } t \geq \bar{t} .
$$

Proof. Let $\varepsilon>0$ and $L \geq 0$ be given by (33). We assume that

$$
\|\bar{\mu}\| \leq \varepsilon \quad \text { and } \quad\|\Gamma\|_{\Omega_{\Theta}} \leq \varepsilon L .
$$

Then, Proposition 5.1 implies that

$$
\left|N^{\bar{\mu}, \Gamma}(t, m)\right| \leq \varepsilon, \quad \text { for } t \geq 0 \text { and } m \in[0,1] .
$$

We define the sequence $\left(\bar{N}_{n}\right)_{n \in \mathbb{N}}$ by

$$
\bar{N}_{0}(t, m)=K\left(t-\tau_{\max }, m\right) N^{\bar{\mu}, \Gamma}\left(\tau_{\max }, \chi\left(-\left(t-\tau_{\max }\right), m\right)\right),
$$

and

$$
\bar{N}_{n}(t, m)=\bar{N}_{0}(t, m)-I_{\tau_{\max }}\left(\bar{N}_{n-1}\right)(t, m)+J_{\tau_{\max }}\left(\bar{N}_{n-1}\right)(t, m),
$$

for $t \geq \tau_{\max }, m \in[0,1]$ and $n \in \mathbb{N}^{*}$, with

$$
I_{\tau_{\max }}(N)(t, m)=\int_{\tau_{\max }}^{t} K(t-s, m) \beta(\chi(-(t-s), m), N(s, \chi(-(t-s), m))) N(s, \chi(-(t-s), m)) d s
$$

and

$$
\begin{aligned}
J_{\tau_{\max }}(N)(t, m)= & \int_{\tau_{\max }}^{t} K(t-s, m) \zeta(\chi(-(t-s), m)) N(s-\tau(\Delta(\chi(-(t-s), m))), \Delta(\chi(-(t-s), m))) \times \\
& \beta(\Delta(\chi(-(t-s), m)), N(s-\tau(\Delta(\chi(-(t-s), m))), \Delta(\chi(-(t-s), m)))) d s,
\end{aligned}
$$

and

$$
\bar{N}_{n}(t, m)=N^{\bar{\mu}, \Gamma}(t, m), \quad \text { for }(t, m) \in\left[0, \tau_{\max }\right] \times[0,1] \text { and } n \in \mathbb{N} .
$$

First, it is easy to check, by using the same reasonning as in the proof of Proposition 5.1, that the sequence $\left(\bar{N}_{n}\right)_{n \in \mathbb{N}}$ satisfies

$$
\left|\bar{N}_{n}(t, m)\right| \leq \varepsilon, \quad \text { for } t \geq 0, m \in[0,1] \text { and } n \in \mathbb{N}^{*},
$$


since

$$
\left|\bar{N}_{0}(t, m)\right| \leq \varepsilon, \quad \text { for } t \geq 0 \text { and } m \in[0,1] .
$$

Secondly, the assumption

$$
L(1+2 \kappa)<\widetilde{\delta}
$$

implies that

$$
L(1+\|\zeta\|)<\widetilde{\delta}
$$

Therefore, there exists $\rho \in(0, \widetilde{\delta})$ such that

$$
L<\frac{\widetilde{\delta}-\rho}{1+\|\zeta\| e^{\rho \tau_{\max }}}<\frac{\widetilde{\delta}}{1+\|\zeta\|} .
$$

Let $t \geq \tau_{\max }$ and $m \in[0,1]$. Then, we get

$$
\left|\bar{N}_{0}(t, m)\right| \leq e^{-\widetilde{\delta}\left(t-\tau_{\max }\right)} \varepsilon \leq e^{-\rho\left(t-\tau_{\max }\right)} \varepsilon \leq \varepsilon .
$$

Since

$$
\left|\bar{N}_{1}(t, m)-\bar{N}_{0}(t, m)\right| \leq\left|I_{\tau_{\max }}\left(\bar{N}_{0}\right)(t, m)\right|+\left|J_{\tau_{\max }}\left(\bar{N}_{0}\right)(t, m)\right|
$$

then, the estimates

$$
\left|I_{\tau_{\max }}\left(\bar{N}_{0}\right)(t, m)\right| \leq \varepsilon L \int_{\tau_{\max }}^{t} e^{-\widetilde{\delta}(t-s)} e^{-\rho\left(s-\tau_{\max }\right)} d s,
$$

and

$$
\left|J_{\tau_{\max }}\left(\bar{N}_{0}\right)(t, m)\right| \leq \varepsilon L\|\zeta\| \int_{\tau_{\max }}^{t} e^{-\widetilde{\delta}(t-s)} e^{-\rho\left(s-2 \tau_{\max }\right)} d s
$$

yield to

$$
\left|\bar{N}_{1}(t, m)-\bar{N}_{0}(t, m)\right| \leq \varepsilon L\left(1+\|\zeta\| e^{\rho \tau_{\max }}\right) e^{-\widetilde{\delta} t} e^{\rho \tau_{\max }} \int_{\tau_{\max }}^{t} e^{(\widetilde{\delta}-\rho) s} d s .
$$

By remarking that

$$
e^{-\widetilde{\delta} t} e^{\rho \tau_{\max }} \int_{\tau_{\max }}^{t} e^{(\widetilde{\delta}-\rho) s} d s \leq \frac{1}{\widetilde{\delta}-\rho} e^{-\rho\left(t-\tau_{\max }\right)},
$$

we obtain

$$
\left|\bar{N}_{1}(t, m)-\bar{N}_{0}(t, m)\right| \leq \varepsilon L \frac{1+\|\zeta\| e^{\rho \tau_{\max }}}{\widetilde{\delta}-\rho} e^{-\rho\left(t-\tau_{\max }\right)} .
$$

It is easy to see by induction, that

$$
\left|\bar{N}_{n}(t, m)-\bar{N}_{n-1}(t, m)\right| \leq \varepsilon\left(L \frac{1+\|\zeta\| e^{\rho \tau_{\max }}}{\widetilde{\delta}-\rho}\right)^{n} e^{-\rho\left(t-\tau_{\max }\right)}, \quad \text { for } n \in \mathbb{N}^{*}
$$

Hence, we get

$$
\begin{aligned}
\left|\bar{N}_{n}(t, m)\right| & \leq \sum_{i=1}^{n}\left|\bar{N}_{i}(t, m)-\bar{N}_{i-1}(t, m)\right|+\left|\bar{N}_{0}(t, m)\right| \\
& \leq \sum_{i=1}^{n} \varepsilon\left(L \frac{1+\|\zeta\| e^{\rho \tau_{\max }}}{\widetilde{\delta}-\rho}\right)^{i} e^{-\rho\left(t-\tau_{\max }\right)}+\varepsilon e^{-\rho\left(t-\tau_{\max }\right)} \\
& \leq \varepsilon e^{-\rho\left(t-\tau_{\max }\right)} \sum_{i=0}^{n}\left(L \frac{1+\|\zeta\| e^{\rho \tau_{\max }}}{\widetilde{\delta}-\rho}\right)^{i}
\end{aligned}
$$


Since

$$
L \frac{1+\|\zeta\| e^{\rho \tau_{\max }}}{\widetilde{\delta}-\rho}<1
$$

then, we finally obtain that

$$
\left|N^{\bar{\mu}, \Gamma}(t, m)\right| \leq \frac{(\widetilde{\delta}-\rho) \varepsilon}{\widetilde{\delta}-\rho-L\left(1+\|\zeta\| e^{\rho \tau_{\max }}\right)} e^{-\rho\left(t-\tau_{\max }\right)}, \quad \text { for all } t \geq \tau_{\max }
$$

Hence, we have obtained the inequality (36) with $\bar{t}=\tau_{\max }, \epsilon=\max \{\varepsilon, \varepsilon L\}, d=\rho$ and

$$
c=\frac{(\widetilde{\delta}-\rho) \varepsilon}{\widetilde{\delta}-\rho-L\left(1+\|\zeta\| e^{\rho \tau_{\max }}\right)} .
$$

This completes the proof.

By using Theorem 5.1, we can prove the local exponential stability of the trivial solution of Problem (26)-(27). This result is presented in the following corollary.

Corollary 5.1. Assume that

$$
L(1+2 \kappa)<\min \{\widetilde{\gamma}, \widetilde{\delta}\} .
$$

Then, the trivial solution of Problem (20)-(27) is locally exponentially stable.

Proof. First, since $L(1+2 \kappa)<\widetilde{\delta}$, then Theorem 5.1 implies that the trivial solution of Equation (26) is locally exponentially stable. Moreover, since we suppose that $L(1+2 \kappa)<\widetilde{\gamma}$, we can show, by using the same arguments as in the proof of Theorem 5.1 and a variation of constant formula for $P$, that the trivial solution of Equation (27) is locally exponentially stable, as soon as the trivial solution of Equation (26) has this property.

Finally, by using Theorem 5.1, we can obtain the global stability of the trivial solution of Problem (26)-(27). This is done in the next proposition.

Proposition 5.2. Assume that the mapping $x \mapsto x \beta(m, x)$ is Lipschitz continuous for all $m \in[0,1]$, with a Lipschitz constant $L$, and that

$$
L(1+2 \kappa)<\min \{\widetilde{\gamma}, \widetilde{\delta}\} .
$$

Let $\Gamma \in C\left(\Omega_{\Theta}\right)$ and $\mu$ be a continuous function such that $\bar{\mu} \in C[0,1]$. If $\|\Gamma\|_{\Omega_{\Theta}} \leq L\|\bar{\mu}\|$, then, the trivial solution of Problem (20)-(2y) is globally exponentially stable.

Proof. We first show, as in the proof of Proposition 5.1, that

$$
\left|N^{\bar{\mu}, \Gamma}(t, m)\right| \leq\|\bar{\mu}\|, \quad \text { for } t \geq 0 \text { and } m \in[0,1] .
$$

Then, by using the same reasonning as in the proofs of Theorem 5.1 and Corollary 5.1, we conclude.

We can give some explanations about the condition $L(1+2 \kappa)<\min \{\widetilde{\gamma}, \widetilde{\delta}\}$. In fact, we can notice that this inequality is satisfied if $\widetilde{\gamma}$ and $\widetilde{\delta}$ are large or if $L$ is small enough. This corresponds, biologically, to the case where the mortality rates $(\gamma$ and $\delta)$ are important or to the case when only a few cells are introduced in the proliferating phase, and then the cells supply is not sufficient ( $L$ is a bound of the number of introduced cells). 


\section{Discussion}

It is usually believed that the function $\beta$ is a Hill function (see Mackey [15]) given by

$$
\beta(m, x)= \begin{cases}\beta_{0}(m) \frac{\theta^{n}(m)}{\theta^{n}(m)+x^{n}}, & \text { for } x \geq 0, m \in[0,1], \\ \beta_{0}(m), & \text { for } x<0, m \in[0,1],\end{cases}
$$

with $\theta$ and $\beta_{0}$ two continuous and positive functions, and $n \geq 1$. In this case, the mapping $x \mapsto x \beta(m, x)$ is always Lipshitz continuous, with a Lipschitz constant equals to

$$
\sup _{m \in[0,1]} \beta_{0}(m)
$$

Hence, the results of Theorem 3.1 and Corollary 3.2 hold.

By the same way, Condition (32) is easily satisfied, because $\beta(m, x)=\beta(m, 0)$ if $x<0$, and, if $x \geq 0$, the function $x \mapsto \beta(m, x)$ is decreasing for all $m$. Hence, the positivity of the solutions is naturally obtained.

As it has already been noticed by Dyson et al. [10], Mackey and Rudnicki [20] and Adimy and PujoMenjouet [3], we can expect to show the influence of the immature cells population (that means, the population with a small maturity) over the entire population. This has also been obtained by Adimy and Crauste [1] for a model with a proliferating phase duration distributed according to a density.

In particular, by using the result of local stability obtained in Section 5, we will certainly be able to prove that the stability (or the instability) of the immature cells population leads to the global stability (or the instability) of the entire population. This has been displayed for the first time by Mackey and Rudnicki [20] in 1999. Since the stem cells population is known to be at the root of the blood production system, then, this behaviour is naturally expected in our model, and it is the purpose of a next work.

\section{References}

[1] M. Adimy and F. Crauste, Un modèle non-linéaire de prolifération cellulaire : extinction des cellules et invariance, C. R. Acad. Paris Ser I 336, 559-564 (2003).

[2] M. Adimy and F. Crauste, Global stability of a partial differential equation with distributed delay due to cellular replication, Nonlinear Analysis 54, 1469-1491 (2003).

[3] M. Adimy and L. Pujo-Menjouet, A singular transport model describing cellular division, C. R. Acad. Sci. Paris Ser. I Math. 332, 12, 1071-1076 (2001).

[4] M. Adimy and L. Pujo-Menjouet, Asymptotic behaviour of a singular transport equation modelling cell division, Dis. Cont. Dyn. Sys. Ser. B 3, 3, 439-456 (2003).

[5] M. Adimy and L. Pujo-Menjouet, A mathematical model describing cellular division with a proliferating phase duration depending on the maturity of cells, accepted in Electron. J. Differ. Equ.

[6] G. Bradford, B. Williams, R. Rossi and I. Bertoncello, Quiescence, cycling, and turnover in the primitive haematopoietic stem cell compartment, Exper. Hematol. 25, 445-453 (1997).

[7] F.J. Burns and I.F. Tannock, On the existence of a $G_{0}$ phase in the cell cycle, Cell. Tissue Kinet. 19, 321-334 (1970).

[8] R. Crabb, J. Losson and M.C. Mackey, Dependence on initial conditions in non local PDE's and heredetary dynamical systems, Proc. Inter. Conf. Nonlin. Anal. 4 (Tampa Bay, de Gruyter, Berlin), 3125-3136 (1996). 
[9] R. Crabb, M.C. Mackey and A. Rey, Propagating fronts, chaos and multistability in a cell replication model, Chaos 6, 477-492 (1996).

[10] J. Dyson, R. Villella-Bressan and G.F. Webb, A singular transport equation modelling a proliferating maturity structured cell population, Can. Appl. Math. Quart. 4, 65-95 (1996).

[11] J. Dyson, R. Villella-Bressan and G.F. Webb, A nonlinear age and maturity structured model of population dynamics. I : Basic theory., J. Math. Anal. Appl. 242, 1, 93-104 (2000).

[12] J. Dyson, R. Villella-Bressan and G.F. Webb, A nonlinear age and maturity structured model of population dynamics. II : Chaos., J. Math. Anal. Appl. 242, 2, 255-270 (2000).

[13] P.C.L John, The cell cycle, London, Cambridge University Press (1981).

[14] Kato N., Positive global solutions for a general model of size-dependent population dynamics, Abstr. Appl. Anal. 5, 3, 191-206 (2000).

[15] M.C. Mackey, Unified hypothesis of the origin of aplastic anaemia and periodic hematopoiesis, Blood 51, 941-956 (1978).

[16] M.C. Mackey and A. Rey, Multistability and boundary layer development in a transport equation with retarded arguments, Can. Appl. Math. Quart. 1, 1-21 (1993).

[17] M.C. Mackey and A. Rey, Transitions and kinematics of reaction-convection fronts in a cell population model, Physica D 80, 120-139 (1995).

[18] M.C. Mackey and A. Rey, Propagation of population pulses and fronts in a cell replication problem : non-locality and dependence on the initial function, Physica D 86, 373-395 (1995).

[19] M.C. Mackey and R. Rudnicki, Global stability in a delayed partial differential equation describing cellular replication, J. Math. Biol. 33, 89-109 (1994).

[20] M.C. Mackey and R. Rudnicki, A new criterion for the global stability of simultaneous cell replication and maturation processes, J. Math. Biol. 38, 195-219 (1999).

[21] J.M Mitchison, The biology of the cell cycle, London, Cambridge University Press (1971).

[22] Sachs L., The molecular control of hemopoiesis and leukomia, C. R. Acad. Sci. Paris 316, $882-891$ (1993).

[23] Travis C.C. and Webb G.F., Existence and stability for partial functionnal differential equations, Trans. Am. Math. Soc. 200, 395-418 (1974).

[24] Webb G.F., Theory of non-linear age-dependent population dynamics, Monographs and Textbook in Pure and Applied Mathematics, 89, New-York Basel: Marcel Dekker Inc., 294p, 1985. 